TRANSACTIONS OF THE

AMERICAN MATHEMATICAL SOCIETY

Volume 361, Number 7, July 2009, Pages 3463-3481

S 0002-9947(09)04911-3

Article electronically published on February 23, 2009

\title{
PRESCRIBING CURVATURES ON THREE DIMENSIONAL RIEMANNIAN MANIFOLDS WITH BOUNDARIES
}

\author{
LEI ZHANG
}

\begin{abstract}
Let $(M, g)$ be a complete three dimensional Riemannian manifold with boundary $\partial M$. Given smooth functions $K(x)>0$ and $c(x)$ defined on $M$ and $\partial M$, respectively, it is natural to ask whether there exist metrics conformal to $g$ so that under these new metrics, $K$ is the scalar curvature and $c$ is the boundary mean curvature. All such metrics can be described by a prescribing curvature equation with a boundary condition. With suitable assumptions on $K, c$ and $(M, g)$ we show that all the solutions of the equation can only blow up at finite points over each compact subset of $\bar{M}$; some of them may appear on $\partial M$. We describe the asymptotic behavior of the blow-up solutions around each blow-up point and derive an energy estimate as a consequence.
\end{abstract}

\section{INTRODUCTION}

In geometric analysis two well-known problems are closely related: the Nirenberg problem (or the Kazdan-Warner problem) and the Yamabe problem. The Nirenberg problem asks what function $K(x)$ on $\mathbb{S}^{n}$ is the scalar curvature of a metric $g$ on $\mathbb{S}^{n}$ conformal to the standard metric $g_{0}$. From another point of view, on any given compact Riemannian manifold $(M, g)$ without boundary, the Yamabe problem, which was solved through the works of Yamabe [36], Trudinger [35], Aubin [3] and Schoen 32 , concerns whether it is possible to deform $g$ conformally to get a new metric with constant scalar curvature. Similar questions can also be asked on general Riemannian manifolds with boundaries: Let $(M, g)$ be a Riemannian manifold with boundary, and let $K \in C^{1}(M)$ and $c \in C^{1}(\partial M)$ be $C^{1}$ functions defined on $M$ and $\partial M$, respectively. Then, is it possible to deform $g$ conformally to another metric $g_{1}$ so that $K$ is the scalar curvature and $c$ is the boundary mean curvature under $g_{1}$ ? By writing $g_{1}$ as $g_{1}=u^{\frac{4}{n-2}} g, K$ and $c$ are related to the scalar curvature $R_{g}$ and the mean curvature $h_{g}$ under the metric $g$ by

$$
\left\{\begin{array}{l}
K=-\frac{4(n-1)}{n-2} u^{-\frac{n+2}{n-2}}\left(\Delta_{g} u-\frac{n-2}{4(n-1)} R_{g} u\right), \\
c=\frac{2}{n-2} u^{-\frac{n}{n-2}}\left(\partial_{\nu_{g}} u+\frac{n-2}{2} h_{g} u\right)
\end{array}\right.
$$

where $\nu_{g}$ is the unit normal vector pointing to the outside of $\partial M . \Delta_{g}$ is the Laplace-Beltrami operator, which can be written as $\Delta_{g}=\frac{1}{\sqrt{g}} \partial_{i}\left(\sqrt{g} g^{i j} \partial_{j}\right)$ in local coordinates.

Received by the editors September 13, 2006.

2000 Mathematics Subject Classification. Primary 35J60, 53B20.

Key words and phrases. Scalar curvature, mean curvature, Harnack inequality.

The author was supported by National Science Foundation Grant 0600275 (0810902).

(C)2009 American Mathematical Society 
If $K$ and $c$ are constants and $(M, g)$ is compact, the existence of a metric with constant scalar curvature and boundary mean curvature is always referred to as the boundary Yamabe problem (BYP). Many cases of the BYP have been solved by Escobar [17, 18, 19, Han and Li [22] and Marques [28]. But unlike the completely solved Yamabe problem, some cases of the BYP are still open. In general, the boundary terms in (1.1) make the nature of equation (1.1) very different from its counterpart without the boundary condition. Some difficulties created by the boundary terms are still not completely understood. In this article, we focus on three dimensional Riemannian manifolds with boundaries and consider the corresponding prescribing curvature equations defined on these manifolds.

Let $(M, g)$ be a smooth three dimensional complete Riemannian manifold with boundary $\partial M$. Suppose $K(x)>0$ and $c(x)$ are $C^{1}$ functions defined on $M$ and $\partial M$, respectively. If $g_{1}=u^{4} g(u>0$ smooth $)$ is a metric conformal to $g$ that takes $K$ as the scalar curvature and $c$ as the mean curvature on $\partial M$, one can write the equation as

$$
\left\{\begin{array}{l}
\Delta_{g} u-\frac{1}{8} R_{g} u+\frac{1}{8} K(x) u^{5}=0, \quad \text { on } M, \\
\partial_{\nu_{g}} u+\frac{1}{2} h_{g} u=\frac{1}{2} c(x) u^{3}, \quad \text { on } \partial M .
\end{array}\right.
$$

The purpose of this article is to understand the bubbling phenomena of (1.2) under natural assumptions on $K$ and $c$. Without the boundary condition, the bubbling phenomenon of equation (1.2) and its high dimensional variants have been studied extensively under various assumptions. The reader is referred to the following incomplete list and the references therein 4, 5, 6, 7, 11, 12, 13, 15, 16, 20, 21, 23, 24, 25, 26, 27, 28, 31, 32, 37. However, much less work can be found to address the case with boundary conditions. In this article we describe the blow-up phenomenon for (1.2) under weak assumptions on $K$ and $c$.

To state our main result, we first remark that $M$ may not be compact, so our result concerns the blow-up phenomenon for every compact subset of $M$ that shares with $M$ a part of its boundary. Namely, let $V$ be a subset of $M$ such that $\bar{V}$ is compact and let $\Gamma=\partial V \cap \partial M$ be the part of the boundary that $V$ shares with $M$. Then our main result can be stated as follows:

Theorem 1.1. Let $(M, g), R_{g}, h_{g}, V, \Gamma, K, c$ be described as above. Let $u>0$ be a classical solution of (1.2). Suppose $\Gamma$ is umbilic and there exists $\epsilon_{0}>0$ such that for every point $p \in \Gamma, g$ is conformal to the Euclidean metric $\delta$ in $B\left(p, \epsilon_{0}\right) \cap M$. Assume in addition that for some $\Lambda>0$,

$$
\begin{gathered}
\frac{1}{\Lambda} \leq K(x) \leq \Lambda, \\
\max \left(\|\nabla K(x)\|_{L^{\infty}(M)},\|c\|_{C^{1}(\partial M)}\right) \leq \Lambda .
\end{gathered}
$$

Then for some $C\left(M, g, \Lambda, V, \epsilon_{0}\right)>0$ and integer $m\left(M, g, \Lambda, V, \epsilon_{0}\right)>0$, there exist local maximum points of $u$, denoted as $S:=\left\{P_{1}, \ldots, P_{m}\right\}$, such that

$$
\begin{gathered}
\operatorname{dist}_{g}\left(P_{i}, P_{j}\right) \geq \frac{1}{C}, \quad \frac{1}{C} u\left(P_{i}\right) \leq u\left(P_{j}\right) \leq C u\left(P_{i}\right), \quad \forall i \neq j, \\
\frac{1}{C} \sum_{l=1}^{m} \xi_{P_{l}, u\left(P_{l}\right)^{2}} \leq u \leq C \sum_{l=1}^{m} \xi_{P_{l}, u\left(P_{l}\right)^{2}}, \quad \text { on } \quad \bar{V},
\end{gathered}
$$


where

$$
\xi_{Q, \mu}(P):=\left(\frac{\mu}{1+\mu^{2} \operatorname{dist}(P, Q)^{2}}\right)^{\frac{1}{2}}, \quad P \in \bar{V} .
$$

As a consequence, we have the following energy estimate:

Corollary 1.1. Under the assumptions of Theorem 1.1, we have

$$
\int_{V}\left(\left|\nabla_{g} u\right|^{2}+u^{6}\right) \leq C\left(M, g, \Lambda, V, \epsilon_{0}\right) .
$$

If $(M, g)$ is compact, Theorem 1.1 concludes that if the metric is locally conformally flat near $\partial M$, which is umbilic, then there are only a finite number of blow-up points in $\bar{M}$ and the solutions of (1.2) can be estimated by (1.3).

The results of Theorem 1.1 and Corollary 1.1 are closely related to a priori estimates of the solutions. When $(M, g)$ is compact with a boundary and $K$ and $c$ are constants, various compactness results and related discussions can be found in [2], 20, 22], 30] and the reference therein. The $C^{1}$ assumptions on $K$ and $c$ in Theorem 1.1 should be sharp. The reason is that even for the interior equations the $C^{1}$ assumption on $K$ is necessary to have a description of the blow-up phenomenon by (1.3). So Theorem 1.1 implies that for this three dimensional situation, the $C^{1}$ assumption on $c$ does not lead to more restrictions on $K$. For dimensions 4 and higher, we expect the situation to be much more subtle: first the assumption on $K$ will be more delicate and should be made in neighborhoods of its critical points; then the sign of $c$ at the blow-up point will make a more significant difference. Also, the flatness assumptions in the neighborhood of the critical points of $K$ and $c$ might be related. As the dimension grows higher, the relations will become more subtle and new phenomena will come out.

The major step in the proof of Theorem 1.1 is to establish a Harnack type inequality near $\Gamma$. This is done by the well-known method of moving planes. Unlike most of the previous works on this type of Harnack inequality established only for the interior equations, the equation in Theorem 1.1 has new features that were not dealt with before. Generally speaking, the method of moving planes requires a delicate construction of some test functions. Now this becomes more difficult for two reasons: first, the symmetry of the domain is destroyed; second, when blowup happens on the boundary or close to the boundary, the function $c$ creates more error terms for the test functions to control. In this article we found a way to handle all the difficulties for dimension three; our approach is motivated by Caffarelli's magnificent ideas in [9], 10] on free boundary problems.

In the next few sections, we mainly focus on the proof of the Harnack type inequality on upper half Euclidean balls and we shall mention how to obtain the interior estimate away from the boundary $\Gamma$.

\section{HARNACK TYPE INEQUALITIES ON THE BOUNDARY}

The proof of Theorem 1.1 can be divided into two parts. First we consider the region close to $\Gamma: N\left(\Gamma, \epsilon_{0}\right)$, i.e. the $\epsilon_{0}$ neighborhood of $\Gamma$. In this region we shall establish a Harnack type inequality. Then outside this region, we use the techniques developed in 25] to get the same type of Harnack inequality. Note that for this interior part we don't need to assume the metric to be locally conformally flat.

Since $(M, g)$ is locally conformally flat near $\Gamma$, at each point $p \in \Gamma$ we can find a positive smooth function $\phi$ such that $\delta=\phi^{4} g$, where $\delta$ is the Euclidean metric. The 
umbilicity of $\Gamma$ implies that $\partial M$ near $p$ is either a piece of a sphere or a hyperplane in the new metric. Since both neighborhoods are conformally equivalent through the inversion map $f(x)=x|x|^{-2}$, we just assume that near $p$ the boundary is a hyperplane. Then the equation in the neighborhood of $p$ can be written as

$$
\begin{cases}\Delta\left(\frac{u}{\phi}\right)+K\left(\frac{u}{\phi}\right)^{5}=0 & B_{\epsilon_{1}}^{+} \subset \mathbb{R}^{3}, \\ \partial_{x_{3}}\left(\frac{u}{\phi}\right)=-c\left(\frac{u}{\phi}\right)^{3} & \text { on } \quad \partial^{\prime} B_{\epsilon_{1}}^{+},\end{cases}
$$

where $\epsilon_{1}>0$ is a positive constant that only depends on $\epsilon_{0}, M, g$, and $\Gamma$. $B_{\epsilon_{1}}^{+}$is the upper half ball of radius $\epsilon_{1}, \partial^{\prime} B_{\epsilon_{1}}^{+}=\partial B_{\epsilon_{1}}^{+} \cap \partial \mathbb{R}_{+}^{3}$. The computation above is based on the following conformal covariant properties of the two operators:

$$
\begin{aligned}
\Delta\left(\frac{u}{\phi}\right) & =\phi^{-5}\left(\Delta_{g} u-c(n) R_{g} u\right), \\
-\partial_{x_{3}}\left(\frac{u}{\phi}\right) & =\phi^{-3}\left(\partial_{\nu_{g}} u+\frac{1}{2} h_{g} u\right) .
\end{aligned}
$$

So the whole equation is reduced to the Euclidean case. From now on in this section we just consider the following case (by abusing the notation we still use $K$ to denote a positive $C^{1}$ function on an upper half disk $B_{3 R}^{+}$and $c$ to denote a $C^{1}$ function defined on $\partial^{\prime} B_{3 R}^{+}$, the lower part of $\left.\partial B_{3 R}^{+}\right)$:

$$
\begin{cases}\Delta u+K(x) u^{5}=0 & B_{3 R}^{+} \subset \mathbb{R}^{3}, \\ \frac{\partial u}{\partial x_{3}}=c\left(x^{\prime}\right) u^{3} & \text { on } \quad \partial^{\prime} B_{3 R}^{+},\end{cases}
$$

where $\partial^{\prime} B_{3 R}^{+}=\partial B_{3 R}^{+} \cap \partial \mathbb{R}_{+}^{3}$, and $x^{\prime}$ is the projection of $x$ to $\partial^{\prime} B_{3 R}^{+}$. The main result of this section is

Proposition 2.1. Let $u \in C^{1}\left(\overline{B_{3 R}^{+}}\right) \cap C^{2}\left(B_{3 R}^{+}\right)$be a positive function that solves (2.1). Assume for some $\Lambda>0$ that $K$ and $c$ satisfy

$$
\frac{1}{\Lambda} \leq K(x) \leq \Lambda, \quad|\nabla K(x)| \leq \Lambda R^{-1}, \forall x \in \overline{B_{3 R}^{+}}
$$

and

$$
\left|c\left(x^{\prime}\right)\right| \leq \Lambda, \quad\left|\nabla c\left(x^{\prime}\right)\right| \leq \Lambda R^{-1}, \quad \forall x^{\prime} \in \partial^{\prime} B_{3 R}^{+}
$$

respectively. Then for some $C(\Lambda)>0$,

$$
\max _{\bar{B}_{R}^{+}} u \cdot \min _{\bar{B}_{2 R}^{+}} u \leq C(\Lambda) R^{-1} .
$$

This Harnack type inequality reveals important information about the interaction of bubbles (large local maximum points) of $u$, which can be seen in the following.

Corollary 2.1. Under the assumptions in Theorem 2.1, there exist a positive constant $C(\Lambda)$, a positive integer $m(\Lambda)$, and a set of $m$ local maximum points of $u$ in $\bar{B}_{R}^{+}$, denoted as $S:=\left\{P_{1}, \ldots, P_{m}\right\}$, such that

$$
\begin{gathered}
\operatorname{dist}\left(P_{i}, P_{j}\right) \geq R / C, \quad \frac{1}{C} u\left(P_{j}\right) \leq u\left(P_{i}\right) \leq C u\left(P_{j}\right), \quad \forall i \neq j, \\
\frac{1}{C} \sum_{l=1}^{m} \xi_{P_{l}, u\left(P_{l}\right)^{2}} \leq u \leq C \sum_{l=1}^{m} \xi_{P_{l}, u\left(P_{l}\right)^{2}}, \quad \text { on } \quad \bar{B}_{R}^{+},
\end{gathered}
$$


where

$$
\xi_{Q, \mu}(P):=\left(\frac{\mu}{1+\mu^{2} \operatorname{dist}(P, Q)^{2}}\right)^{\frac{1}{2}}, \quad P \in \bar{B}_{R}^{+}
$$

Consequently u satisfies

$$
\int_{B_{R}^{+}}\left(|\nabla u|^{2}+u^{6}\right) \leq C(\Lambda) .
$$

In the proof of Proposition 2.1 we shall omit a selection process of Schoen since it is well known to experts. We only include it in the appendix. At the end of this section we indicate the outline of the proof of Corollary 2.1 based on Proposition 2.1. The proof can be found in 24 with obvious changes, but we indicate the key points for the convenience of the readers.

2.1. The proof of Proposition 2.1. We only need to consider the case $R=1$. The general case can be reduced to the case $R=1$ by considering the function $R^{\frac{1}{2}} u(R \cdot)$. The proof is by a contradiction. Suppose (2.4) does not hold. Then there exists a sequence $u_{i}\left(x_{i}\right)$ such that

$$
u_{i}\left(x_{i}\right) \min _{\bar{B}_{2}^{+}} u_{i} \geq i,
$$

where $u_{i}\left(x_{i}\right)=\max _{\bar{B}_{1}^{+}} u_{i}$ and $x_{i} \in \bar{B}_{1}$. Clearly the above inequality implies $u_{i}\left(x_{i}\right) \rightarrow \infty$. By a standard selection process of Schoen 31 and the classification theorems of Caffarelli-Gidas-Spruck [ 8 ] and Li and Zhu [27, one can consider $x_{i}$ as a local maximum of $u_{i}$. Moreover the following sequence of functions

$$
\bar{v}_{i}(y):=u_{i}\left(x_{i}\right)^{-1} u_{i}\left(u_{i}\left(x_{i}\right)^{-2} y+x_{i}\right)
$$

converges in $C^{2}$ over any finite domain in the following two cases:

(1) If $\lim _{i \rightarrow \infty} u_{i}^{2}\left(x_{i}\right) x_{i 3} \rightarrow \infty$, the $\bar{v}_{i}(y)$ converge in $C_{l o c}^{2}\left(\mathbb{R}^{3}\right)$ to $U(y)$ which satisfies

$$
\left\{\begin{array}{l}
\Delta U(y)+\lim _{i \rightarrow \infty} K_{i}\left(x_{i}\right) U(y)^{5}=0, \quad \text { on } \mathbb{R}^{3}, \\
U(0)=1=\max _{\mathbb{R}^{3}} U .
\end{array}\right.
$$

(2) If $\lim _{i \rightarrow \infty} u_{i}^{2}\left(x_{i}\right) x_{i 3}$ is bounded, by passing to a subsequence we assume

$$
\lim _{i \rightarrow \infty} u_{i}^{2}\left(x_{i}\right) x_{i 3}=t_{0} .
$$

In this case the $\bar{v}_{i}$ converge uniformly to $U$ which satisfies

$$
\begin{cases}\Delta U(y)+\lim _{i \rightarrow \infty} K_{i}\left(x_{i}\right) U(y)^{5}=0, & y_{3}>t_{0}, \\ \partial_{3} U(y)=\lim _{i \rightarrow \infty} c_{i}\left(x_{i}^{\prime}\right) U^{3}, & y_{3}=-t_{0} \\ U(0)=1=\max U & \end{cases}
$$

Since the selection process and the application of the classification theorems are standard, we put the details in the Appendix. Similar techniques can also be found in [11, 24], 37, etc.

The proof of Proposition 2.1 that follows can be divided into three steps. First we rule out case one; i.e., we shall show that the blow-up points cannot be far away from the boundary. In the second step we prove the case of $\lim _{i \rightarrow \infty} c\left(x_{i}^{\prime}\right) \leq 0$. Then in the final step we prove the case of $\lim _{i \rightarrow \infty} c\left(x_{i}^{\prime}\right)>0$. 
2.1.1. Step one. In this subsection we derive a contradiction to

$$
\lim _{i \rightarrow \infty} T_{i}:=\lim _{i \rightarrow \infty} u_{i}^{2}\left(x_{i}\right) x_{i 3} \rightarrow \infty .
$$

With no loss of generality we assume $\lim _{i \rightarrow \infty} K_{i}\left(x_{i}\right)=3$. In this case, the $U$ in (2.9) is of the form

$$
U(y)=\left(1+|y|^{2}\right)^{-\frac{1}{2}}
$$

Let $M_{i}=u_{i}\left(x_{i}\right)$. In this subsection we use $v_{i}$ instead of $\bar{v}_{i}$, i.e.

$$
v_{i}(y)=M_{i}^{-1} u_{i}\left(M_{i}^{-2} y+x_{i}\right) .
$$

The selection process implies $B\left(x_{i}, \frac{1}{2}\right) \cap \mathbb{R}_{+}^{3} \subset B_{2}^{+}$, so the rescaled domain for $v_{i}$ will have a part of the boundary (the upper part) whose distance to 0 is comparable to $M_{i}^{2}$. By (2.7) we know on this part of the boundary that $v_{i}(y)|y|>i$. By choosing $\epsilon_{i} \rightarrow 0$ slowly we can still obtain $v_{i}(y)|y|>\sqrt{i}$ for $|y|=\epsilon_{i} M_{i}^{2}$. So we define

$$
\Omega_{i}=\left\{y: M_{i}^{-2} y+x_{i} \in B\left(x_{i}, \epsilon_{i}\right) \cap \mathbb{R}_{+}^{3}\right\}
$$

and

$$
\partial^{\prime} \Omega_{i}=\partial \Omega_{i} \cap\left\{y_{3}=-T_{i}\right\}, \quad \partial^{\prime \prime} \Omega_{i}=\partial \Omega_{i} \backslash \partial^{\prime} \Omega_{i} .
$$

Then $v_{i}$ satisfies

$$
\left\{\begin{array}{l}
\Delta v_{i}(y)+K_{i}\left(M_{i}^{-2} y+x_{i}\right) v_{i}(y)^{5}=0, \quad y \in \Omega_{i}, \\
\partial_{3} v_{i}(y) \leq \Lambda v_{i}^{3} \quad y \in \partial \Omega_{i} \cap\left\{y_{3}=-T_{i}\right\}, \quad T_{i}:=M_{i}^{2} x_{i 3} .
\end{array}\right.
$$

Note that in this case we don't need the specific equation for $v_{i}$ on $\partial^{\prime} \Omega_{i}$.

By the discussion on the behavior of $v_{i}$ on $\partial^{\prime \prime} \Omega_{i}$ we have

$$
v_{i}(y)|y| \rightarrow \infty \quad \text { for } \quad y \in \partial^{\prime \prime} \Omega_{i} .
$$

Let $\lambda \in\left[\frac{1}{2}, 2\right]$ and

Then a direct computation gives

$$
v_{i}^{\lambda}(y):=\left(\frac{\lambda}{|y|}\right) v_{i}\left(\frac{\lambda^{2} y}{|y|^{2}}\right) .
$$

$$
\Delta v_{i}^{\lambda}(y)+K_{i}\left(M_{i}^{-2} y^{\lambda}+x_{i}\right) v_{i}^{\lambda}(y)^{5}=0 \quad \text { for }|y|>\lambda / 2 .
$$

Here we use $y^{\lambda}=\lambda^{2} y /|y|^{2}$.

In this step we assume $\lambda \in\left[\frac{1}{2}, 2\right]$. Let $\Sigma_{\lambda}=\Omega_{i} \backslash \bar{B}_{\lambda}$. Note that for simplicity we shall omit $i$ in some notation. Let $w_{\lambda}=v_{i}-v_{i}^{\lambda}$ and consider the equation for $w_{\lambda}$ :

$$
\begin{cases}\Delta w_{\lambda}+b_{\lambda} w_{\lambda}=Q_{\lambda}, & \text { on } \Sigma_{\lambda}, \\ \partial_{3} w_{\lambda} \leq \Lambda \xi_{i} w_{\lambda}+\Lambda\left(v_{i}^{\lambda}\right)^{3}-\partial_{3}\left(v_{i}^{\lambda}\right), & \text { on } \partial^{\prime} \Omega_{i} \backslash \bar{B}_{\lambda},\end{cases}
$$

where $b_{\lambda}$ and $\xi_{i}$ are obtained from the mean value theorem:

$$
\begin{aligned}
& b_{\lambda}(y)=5 K_{i}\left(M_{i}^{-2} y+x_{i}\right) \int_{0}^{1}\left(t v_{i}(y)+(1-t) v_{i}^{\lambda}(y)\right)^{4} d t \\
& \xi_{i}(y)=3 \int_{0}^{1}\left(t v_{i}+(1-t) v_{i}^{\lambda}\right)^{2} d t
\end{aligned}
$$

and

$$
Q_{\lambda}(y)=\left(K_{i}\left(M_{i}^{-2} y^{\lambda}+x_{i}\right)-K_{i}\left(M_{i}^{-2} y+x_{i}\right)\right)\left(v_{i}^{\lambda}(y)\right)^{5}
$$

is estimated as follows:

$$
\left|Q_{\lambda}(y)\right| \leq C_{0}(\Lambda) M_{i}^{-2}|y|^{-4}, \quad \text { on } \Sigma_{\lambda} .
$$


Since $v_{i}$ converges in the $C^{2}$ norm over $U$ over any fixed finite domain, $v_{i}^{\lambda}$ is close to $U^{\lambda}$, the Kelvin transformation of $U$. By direct computation $U>U^{\lambda}$ for $|y|>\lambda$ and $\lambda \in(0,1)$. On the other hand, $U<U^{\lambda}$ for $\lambda>1$ and $|y|>\lambda$. So the strategy of the proof is to find a test function $h_{\lambda}$ ( $i$ is omitted in this notation) so that the moving sphere method works for $w_{\lambda}+h_{\lambda}$, and the $h_{\lambda}$ is just a perturbation of $w_{\lambda}$, which means $h_{\lambda}(y)=\circ(1)|y|^{-1}$ in $\Sigma_{\lambda}$. Then it is possible to move the spheres from a position less than 1 to a position greater than 1 , keeping $w_{\lambda}+h_{\lambda}>0$ in $\Sigma_{\lambda}$. But this is a contradiction since $w_{\lambda}+h_{\lambda}$ converges to $U-U^{\lambda}$ in finite domains and $U<U^{\lambda}$ for $\lambda>1$ and $|y|>\lambda$.

The test function in this section is

$$
h_{\lambda}(y)=C_{0} M_{i}^{-2}\left(-\frac{1}{2}|y|^{-2}+\lambda^{-1}|y|^{-1}-\frac{1}{2} \lambda^{-2}\right),
$$

$h_{\lambda}$ is a radial function, and the function $h_{\lambda}(r)$ satisfies

$$
\left\{\begin{array}{l}
h_{\lambda}^{\prime \prime}(r)+\frac{2}{r} h_{\lambda}^{\prime}(r)=-C_{0} M_{i}^{-2} r^{-4}, \quad r>\lambda \\
h_{\lambda}(\lambda)=h_{\lambda}^{\prime}(\lambda)=0
\end{array}\right.
$$

By the maximum principle, $h_{\lambda}(r)<0$ for $r>\lambda$. Note that the $C_{0}$ in (2.16) is the same as the one in (2.15).

Now we consider the equation for $w_{\lambda}+h_{\lambda}$. From (2.14) we have

$$
\left(\Delta+b_{\lambda}\right)\left(w_{\lambda}+h_{\lambda}\right)=Q_{\lambda}+\Delta h_{\lambda}+b_{\lambda} h_{\lambda} \leq 0 \quad \text { on } \Sigma_{\lambda} .
$$

The reason is that $\Delta h_{\lambda}+Q_{\lambda} \leq 0$ and $h_{\lambda} \leq 0$ in $\Sigma_{\lambda}$.

On $\partial^{\prime} \Omega_{i} \backslash \bar{B}_{\lambda}$ we have

$$
\partial_{3}\left(w_{\lambda}+h_{\lambda}\right) \leq \Lambda \xi_{i}\left(w_{\lambda}+h_{\lambda}\right)+\Lambda\left(v_{i}^{\lambda}\right)^{3}-\partial_{3}\left(v_{i}^{\lambda}\right)+\partial_{3} h_{\lambda}-\Lambda \xi_{i} h_{\lambda} .
$$

By the definition of $h_{\lambda}$ one can verify that $h_{\lambda}=\circ(1)|y|^{-1}$ over $\Sigma_{\lambda}$. Let

$$
O_{\lambda}=\left\{y \in \Sigma_{\lambda} \mid \quad v_{i}(y) \leq 2 v_{i}^{\lambda}(y)\right\} .
$$

Then we observe that the maximum principle in the moving sphere process only needs to be applied over $O_{\lambda}$ because outside $O_{\lambda}, w_{\lambda}+h_{\lambda}>0$. Now we claim that

$$
\Lambda\left(v_{i}^{\lambda}\right)^{3}-\partial_{3}\left(v_{i}^{\lambda}\right)+\partial_{3} h_{\lambda}-\Lambda \xi_{i} h_{\lambda}<0, \quad \text { on } \quad \partial^{\prime} \Omega_{i} \cap \overline{O_{\lambda}} .
$$

Once we have (2.19), (2.18) becomes

$$
\partial_{3}\left(w_{\lambda}+h_{\lambda}\right) \leq \Lambda \xi_{i}\left(w_{\lambda}+h_{\lambda}\right) \quad \text { on } \quad \partial^{\prime} \Omega_{i} \cap \overline{O_{\lambda}},
$$

which is the form for the application of the moving sphere method.

To see (2.19), we first observe that

$$
\begin{aligned}
\partial_{3}\left(v_{i}^{\lambda}\right)= & \lambda|y|^{-3} T_{i} v_{i}\left(y^{\lambda}\right)+2 \lambda^{3}|y|^{-5} \sum_{j=1}^{3} \partial_{j} v_{i}\left(y^{\lambda}\right) y_{j} T_{i} \\
& +\lambda^{3}|y|^{-3} \partial_{3} v_{i}\left(y^{\lambda}\right) \quad \text { on } \quad \partial^{\prime} \Omega_{i} \backslash \bar{B}_{\lambda} .
\end{aligned}
$$

The second term and the third term on the right are negligible compared to the first term on the right. The reason is that $v_{i} \rightarrow U$ in $C_{l o c}^{2}\left(\mathbb{R}^{3}\right)$, which implies

$$
\nabla v_{i}\left(y^{\lambda}\right) \rightarrow \nabla U(0)=0 .
$$

On the other hand, by the assumption $T_{i} \rightarrow \infty$, we see that the first term dominates the other two terms.

Next we see that the assumption $T_{i} \rightarrow \infty$ makes $\left|\partial_{3}\left(v_{i}^{\lambda}\right)\right|$ dominate $\Lambda\left(v_{i}^{\lambda}\right)^{3}$ as the latter is of the order $O\left(|y|^{-3}\right)$. By the definition of $h_{\lambda}$ and the definition of the 
domain $\Omega_{i}$, we see easily that $\left|\partial_{3}\left(v_{i}^{\lambda}\right)\right|$ also dominates $\partial_{3} h_{\lambda}$. Finally in $\bar{O}_{\lambda}$, $\xi_{i}$ is comparable to $|y|^{-2}$, so $\xi_{i} h_{\lambda}$ is comparable to $O\left(M_{i}^{-2}|y|^{-2}\right)$, which is much smaller than $\left|\partial_{3}\left(v_{i}^{\lambda}\right)\right|$. (2.19) is proved.

The process of making the moving sphere process start is standard, even though the boundary condition makes it different from the interior case.

Lemma 2.1. For any fixed $\lambda_{0} \in\left(\frac{1}{2}, 1\right)$ and all large $i$,

$$
w_{\lambda_{0}}+h_{\lambda_{0}}>0 \text { in } \Sigma_{\lambda_{0}} \text {. }
$$

Proof of Lemma 2.1. Since $v_{i} \rightarrow U$ in $C_{\text {loc }}^{2}\left(\mathbb{R}^{3}\right)$ and $U>U^{\lambda}$ for $|y|>\lambda$ if $\lambda \in\left(\frac{1}{2}, 1\right)$, by the convergence of $v_{i}$ to $U$ and the fact $h_{\lambda}=\circ(1)$ over finite domains we can check easily that for any fixed $R_{1} \gg 1$,

$$
w_{\lambda_{0}}+h_{\lambda_{0}}>0 \text { in } \Sigma_{\lambda_{0}} \cap B_{R_{1}} .
$$

Since $R_{1}$ is chosen sufficiently large, one can find $\sigma\left(\lambda_{0}\right)>0$ such that

$$
v_{i}^{\lambda_{0}}(y) \leq(1-3 \sigma)|y|^{-1} \quad|y| \geq R_{1}
$$

and

$$
v_{i}(y)>(1-\sigma)|y|^{-1} \quad \text { on } \quad|y|=R_{1} .
$$

The definitions of $h_{\lambda_{0}}$ and $\Omega_{i}$ imply $\left|h_{\lambda_{0}}(y)\right| \leq \frac{\sigma}{5}|y|^{-1}$ for $|y|>R_{1}$. So to finish the proof of this lemma it is enough to show

$$
v_{i}(y)>(1-2 \sigma)|y|^{-1} \quad \text { in } \quad \Omega_{i} \backslash \overline{B_{R_{1}}} .
$$

On $|y|=R_{1}$ and $\partial^{\prime \prime} \Omega_{i}$ we certainly have $v_{i}>(1-2 \sigma)|y|^{-1}$. To apply the maximum principle to the superharmonic function $v_{i}-(1-2 \sigma)|y|^{-1}$, we need

$$
\partial_{3}\left(v_{i}-(1-2 \sigma)|y|^{-1}\right) \leq \Lambda\left(v_{i}^{3}-(1-2 \sigma)^{3}|y|^{-3}\right) \quad \text { on } \partial^{\prime} \Omega_{i} .
$$

Using $T_{i} \rightarrow \infty$ we obtain by direct computation that

$$
\partial_{3}\left((1-2 \sigma)|y|^{-1}\right)>\Lambda\left((1-2 \sigma)|y|^{-1}\right)^{3} \quad \text { on } \partial^{\prime} \Omega_{i} .
$$

(2.22) follows immediately. (2.22) means that the non-positive minimum cannot be attained on $\partial^{\prime} \Omega_{i}$. Lemma 2.1 is established.

Lemma 2.1 means that the moving sphere process can start at $\lambda=\lambda_{0}$. The purpose of the moving spheres is to show that

$$
w_{\lambda}+h_{\lambda}>0 \text { in } \Sigma_{\lambda} \text { for all } \lambda \in\left[\lambda_{0}, \lambda_{1}\right] .
$$

Once this is proved, we have $U>U^{\lambda_{1}}$ for some $|y|>\lambda_{1}$, which is a contradiction to the fact that $\lambda_{1}>1$. To see why (2.23) holds, first, (2.17) and (2.20) mean that the maximum principle holds for $w_{\lambda}+h_{\lambda}$, and second, (2.12) means that there is no touch on $\partial^{\prime \prime} \Omega_{i}$. Step one is established.

2.1.2. Step two. In this step, we deal with the case $\lim _{i \rightarrow \infty} c_{i}\left(x_{i}^{\prime}\right) \leq 0$. Let

$$
-c_{0}=\lim _{i \rightarrow \infty} c_{i}\left(x_{i}^{\prime}\right) \leq 0 .
$$

In this case the limit of $\bar{v}_{i}$ is $U$, which satisfies

$$
\left\{\begin{array}{l}
\Delta U+3 U^{5}=0, \quad y \in \mathbb{R}^{3}, y_{3}>-\lim _{i \rightarrow \infty} T_{i}, \\
\partial_{3} U=-c_{0} U^{3}, \quad y_{3}=-\lim _{i \rightarrow \infty} T_{i}, \\
U(0)=1=\max U
\end{array}\right.
$$

By Li and Zhu's classification theorem [27], $\lim _{i \rightarrow \infty} T_{i}=0$. So we define $v_{i}$ as

$$
v_{i}(y)=M_{i}^{-1} u_{i}\left(M_{i}^{-2} y+x_{i}^{\prime}\right) \text {, }
$$


where $x_{i}^{\prime}$ is the projection of $x_{i}$ on $\partial \mathbb{R}_{+}^{3}$. Then $v_{i} \rightarrow U$ in $C_{l o c}^{2}\left(\overline{\mathbb{R}}_{+}^{3}\right)$. Since $U(0)=1$, we know, from Li and Zhu's theorem, that

$$
U(y)=\left(1+c_{0}^{2}\right)^{\frac{1}{2}}\left(1+\left(1+c_{0}^{2}\right)^{2}\left|y^{\prime}\right|^{2}+\left(1+c_{0}^{2}\right)\left(y_{3}+\frac{c_{0}}{1+c_{0}^{2}}\right)^{2}\right)^{-\frac{1}{2}} .
$$

Let $v_{i}^{\lambda}(y)=\left(\frac{\lambda}{|y|}\right) v_{i}\left(y^{\lambda}\right)$. In this step we let

$$
\lambda_{0}=\frac{1}{2}\left(1+c_{0}^{2}\right)^{-\frac{1}{2}}, \quad \lambda_{1}=2\left(1+c_{0}^{2}\right)^{-\frac{1}{2}}
$$

and we require $\lambda \in\left[\lambda_{0}, \lambda_{1}\right]$ and the moving sphere method will be applied on $\Sigma_{\lambda}=B^{+}\left(0, \epsilon_{i} M_{i}^{2}\right) \backslash \bar{B}_{\lambda}$. A direct computation gives

$$
\left\{\begin{array}{l}
\Delta v_{i}^{\lambda}(y)+K_{i}\left(M_{i}^{-2} y^{\lambda}+x_{i}^{\prime}\right)\left(v_{i}^{\lambda}(y)\right)^{5}=0 \quad \text { on } \Sigma_{\lambda}, \\
\partial_{3} v_{i}^{\lambda}(y)=c_{i}\left(M_{i}^{-2} y^{\lambda}+x_{i}^{\prime}\right)\left(v_{i}^{\lambda}(y)\right)^{3} \quad \text { on } \partial \Sigma_{\lambda} \cap \partial \mathbb{R}_{+}^{3} .
\end{array}\right.
$$

Also by a direct computation one can verify that

$$
\left\{\begin{array}{lll}
U(y)>U^{\lambda}(y), & |y|>\lambda, & 0<\lambda<\left(1+c_{0}^{2}\right)^{-\frac{1}{2}}, \\
U(y)<U^{\lambda}(y), & |y|>\lambda, & \lambda>\left(1+c_{0}^{2}\right)^{-\frac{1}{2}}
\end{array}\right.
$$

For this reason we require $\lambda \in\left[\lambda_{0}, \lambda_{1}\right]$ in this step. The equation for $w_{\lambda}=v_{i}-v_{i}^{\lambda}$ becomes

$$
\begin{cases}\Delta w_{\lambda}+K_{i}\left(M_{i}^{-2} \cdot+x_{i}^{\prime}\right) \xi_{1} w_{\lambda}=Q_{1} & \text { on } \Sigma_{\lambda}, \\ \partial_{3} w_{\lambda}=c_{i}\left(M_{i}^{-2} \cdot+x_{i}^{\prime}\right) \xi_{2} w_{\lambda}+Q_{2} & \text { on } \partial \Sigma_{\lambda} \cap \partial \mathbb{R}_{+}^{3},\end{cases}
$$

where $\xi_{1}$ and $\xi_{2}$ are obtained by the mean value theorem:

$$
\xi_{1}=5 \int_{0}^{1}\left(t v_{i}+(1-t) v_{i}^{\lambda}\right)^{4} d t, \quad \xi_{2}=3 \int_{0}^{1}\left(t v_{i}+(1-t) v_{i}^{\lambda}\right)^{2} d t
$$

and $Q_{1}$ and $Q_{2}$ are error terms to be controlled by test functions:

$$
\begin{aligned}
& Q_{1}(y)=\left(K_{i}\left(M_{i}^{-2} y^{\lambda}+x_{i}^{\prime}\right)-K_{i}\left(M_{i}^{-2} y+x_{i}^{\prime}\right)\right) v_{i}^{\lambda}(y)^{5} \quad \text { on } \Sigma_{\lambda}, \\
& Q_{2}(y)=\left(c_{i}\left(M_{i}^{-2} y+x_{i}^{\prime}\right)-c_{i}\left(M_{i}^{-2} y^{\lambda}+x_{i}^{\prime}\right)\right) v_{i}^{\lambda}(y)^{3} \quad \text { on } \partial \Sigma_{\lambda} \cap \partial \mathbb{R}_{+}^{3} .
\end{aligned}
$$

For $Q_{1}$ and $Q_{2}$ we use

$$
\begin{aligned}
& \left|Q_{1}(y)\right| \leq C_{2}(\Lambda) M_{i}^{-2}|y|^{-4} \quad \text { on } \Sigma_{\lambda}, \\
& \left|Q_{2}(y)\right| \leq C_{2}(\Lambda) M_{i}^{-2}|y|^{-2}\left(1-\frac{\lambda}{|y|}\right) \quad \text { on } \partial \Sigma_{\lambda} \cap \partial \mathbb{R}_{+}^{3} .
\end{aligned}
$$

Note that in $Q_{2}$ it is important to have the $1-\frac{\lambda}{|y|}$ term, even though we don't need this term to appear in the estimate of $Q_{1}$.

The construction of the test function here consists of two parts, $h_{1}$ and $h_{2}$. We first define

$$
h_{1}(r)=C_{3}(\Lambda)\left(r^{-\frac{1}{2}}-\frac{1}{2} \lambda^{\frac{1}{2}} r^{-1}-\frac{1}{2} \lambda^{-\frac{1}{2}}\right) M_{i}^{-2},
$$

where $C_{3}(\Lambda) \gg C_{2}$ is to be determined. $h_{1}(r)$ is the solution of

$$
\left\{\begin{array}{l}
h_{1}^{\prime \prime}(r)+\frac{2}{r} h_{1}^{\prime}(r)=-\frac{C_{3}}{4} M_{i}^{-2} r^{-\frac{5}{2}}, \quad r>\lambda \\
h_{1}(\lambda)=h_{1}^{\prime}(\lambda)=0
\end{array}\right.
$$


By the maximum principle, $h_{1}(r)<0$ for $r>\lambda$. $h_{1}(|y|)$ is the first part of the test function $h_{\lambda}$. To define the second part of the test function, we let $\phi:[1, \infty) \rightarrow$ $[0, \infty)$ be a smooth non-negative function that satisfies

$$
\left\{\begin{array}{l}
\phi(1)=0, \quad \phi(r)=r^{-2} \quad \text { for } \quad r \geq 2 \\
\phi^{\prime}(1)>0, \quad \phi(r)>0 \quad \text { for } \quad r>1
\end{array}\right.
$$

Let $\phi_{\lambda}(\cdot)=\phi(\lambda \cdot)$ and define

$$
h_{2}(y)=-C_{4}(\Lambda) M_{i}^{-2} y_{3} \phi_{\lambda}(|y|),
$$

where $C_{4}(\Lambda)$ is chosen so large that

$$
C_{4} \phi_{\lambda}(|y|)>2 C_{2}|y|^{-2}\left(1-\frac{\lambda}{|y|}\right), \quad|y|>\lambda .
$$

Note that on $\partial^{\prime} \Sigma_{\lambda}:=\partial \Sigma_{\lambda} \cap \partial \mathbb{R}_{+}^{3}$,

$$
\partial_{3} h_{2}(y)=-C_{4} M_{i}^{-2} \phi_{\lambda}(|y|), \quad \partial_{3} h_{1}(y)=0 \quad \text { on } \partial^{\prime} \Sigma_{\lambda} .
$$

Letting $h_{\lambda}=h_{1}+h_{2}$, the equation for $w_{\lambda}+h_{\lambda}$ is

$$
\begin{gathered}
\left(\Delta+K_{i}\left(M_{i}^{-2} \cdot+x_{i}^{\prime}\right) \xi_{1}\right)\left(w_{\lambda}+h_{\lambda}\right)=Q_{1}+\Delta h_{\lambda}+K_{i}\left(M_{i}^{-2} \cdot+x_{i}^{\prime}\right) \xi_{1} h_{\lambda} \quad \text { on } \Sigma_{\lambda}, \\
\left(\partial_{3}-c_{i}\left(M_{i}^{-2} \cdot+x_{i}^{\prime}\right) \xi_{2}\right)\left(w_{\lambda}+h_{\lambda}\right)=-c_{i}\left(M_{i}^{-2} \cdot+x_{i}^{\prime}\right) \xi_{2} h_{\lambda}+\partial_{3} h_{2}+Q_{2} \quad \text { on } \partial^{\prime} \Sigma_{\lambda} .
\end{gathered}
$$

For the application of the moving sphere method we show that the right-hand sides of the above are non-positive. Namely we shall show that

$$
Q_{1}+\Delta h_{1}+\Delta h_{2}+K_{i}\left(M_{i}^{-2} \cdot+x_{i}^{\prime}\right) \xi_{1} h_{\lambda} \leq 0 \quad \text { on } \Sigma_{\lambda}
$$

and

$$
-c_{i}\left(M_{i}^{-2} \cdot+x_{i}^{\prime}\right) \xi_{2} h_{\lambda}+\partial_{3} h_{2}+Q_{2} \leq 0 \quad \text { on } \partial^{\prime} \Sigma_{\lambda} \cap \overline{O_{\lambda}},
$$

where $O_{\lambda}=\left\{y \in \Sigma_{\lambda} \mid v_{i}(y) \leq 2 v_{i}^{\lambda}(y)\right\}$ is the only place where the maximum principle needs to hold, because by the definition of $h_{\lambda}, h_{\lambda}(y)=\circ(1)|y|^{-1}$ in $\Sigma_{\lambda}$, which means $w_{\lambda}+h_{\lambda}>0$ in $\Sigma_{\lambda} \backslash \overline{O_{\lambda}}$.

To see (2.32), first by (2.27) and (2.28), one sees that if $C_{3}$ is large enough, then

$$
\Delta h_{1}+Q_{1} \leq-\frac{1}{8} C_{3} M_{i}^{-2}|y|^{-2.5} \quad \text { on } \Sigma_{\lambda} .
$$

For $h_{2}$ we have

$$
\Delta h_{2}=-C_{4} M_{i}^{-2}\left(\phi_{\lambda}^{\prime \prime}(|y|)+\frac{4}{|y|} \phi_{\lambda}^{\prime}(|y|)\right) y_{3} \quad \text { on } \Sigma_{\lambda} .
$$

So by choosing $C_{3}$ larger if necessary we have

$$
\Delta\left(h_{1}+h_{2}\right)+Q_{1} \leq-\frac{1}{16} C_{3} M_{i}^{-2}|y|^{-2.5} \quad \text { on } \Sigma_{\lambda} .
$$

The term $K_{i}\left(M_{i}^{-2} \cdot+x_{i}\right) \xi_{1} h_{\lambda}$ is non-positive. So (2.32) is proved.

To see (2.33), first by the definition of $h_{2}$ we have

$$
\partial_{3} h_{2}+Q_{2}<-\frac{C_{4}}{2} M_{i}^{-2} \phi_{\lambda}(|y|) \quad \text { on } \partial^{\prime} \Sigma_{\lambda} .
$$


Since $\lim _{i \rightarrow \infty} c_{i}\left(x_{i}^{\prime}\right)=-c_{0} \leq 0$, one uses the fact that $|y| \leq \epsilon_{i} M_{i}^{2}$ and the uniform bound of the $C_{1}$ norm of $c_{i}$ to get

$$
\begin{aligned}
- & c_{i}\left(M_{i}^{-2} \cdot+x_{i}^{\prime}\right) \xi_{2} h_{\lambda} \\
& \leq \circ(1) \xi_{2}\left|h_{1}+h_{2}\right| \leq \circ(1) M_{i}^{-2}|y|^{-2}\left(1-\frac{\lambda}{|y|}\right) \quad \text { on } \partial^{\prime} \Sigma_{\lambda} \cap \bar{O}_{\lambda} .
\end{aligned}
$$

The second inequality above is because in $\bar{O}_{\lambda}, \xi_{2} \sim|y|^{-2}$. We also use $h_{\lambda}(\lambda)=0$ in the above. Then (2.33) follows immediately.

Next we show that the moving sphere process can get started. Namely we have

Lemma 2.2. $w_{\lambda_{0}}+h_{\lambda_{0}}>0$ in $\Sigma_{\lambda_{0}}$ for all large $i$.

Proof of Lemma 2.2. The proof is similar to the one in step one. For $|y| \leq R_{1}$ $\left(R_{1} \gg 1\right), w_{\lambda_{0}}+h_{\lambda_{0}}>0$ is guaranteed by the expressions of $U, U^{\lambda_{0}}$ and the convergence of $v_{i}$ to $U$ over finite domains. Also $h_{\lambda_{0}}=\circ(1)|y|^{-1}$ means $h_{\lambda_{0}}$ is just a perturbation over finite domains. Moreover on $|y|=R_{1}$, there is $\epsilon_{0}>0$ such that

$$
U(y)-U^{\lambda_{0}}(y)>5 \epsilon_{0}\left|y-e_{3}\right|^{-1}, \quad|y|=R_{1}, \quad e_{3}=(0,0,1) .
$$

Also we have

$$
v_{i}^{\lambda_{0}}(y) \leq\left(1-4 \epsilon_{0}\right)\left(1+c_{0}^{2}\right)^{-\frac{1}{2}}\left|y-e_{3}\right|^{-1}, \quad|y|>R_{1}, y \in \Sigma_{\lambda_{0}}
$$

and

$$
\left|U^{\lambda_{0}}(y)-v_{i}^{\lambda_{0}}(y)\right| \leq \epsilon_{0}\left|y-e_{3}\right|^{-1}, \quad|y|>R_{1}
$$

for all $i$ large. So for $y \in \Sigma_{\lambda} \backslash B_{R_{1}}$ we only need to show that

$$
v_{i}(y)>\left(1-\epsilon_{0}\right)\left(1+c_{0}^{2}\right)^{-\frac{1}{2}}\left|y-e_{3}\right|^{-1}, \quad y \in \Sigma_{\lambda} \backslash B_{R_{1}} .
$$

Since $v_{i}(y)-\left(1-\epsilon_{0}\right)\left(1+c_{0}^{2}\right)^{-\frac{1}{2}}\left|y-e_{3}\right|^{-1}$ is a superharmonic function in $\Sigma_{\lambda} \backslash B_{R_{1}}$ and is positive on $|y|=R_{1}$ and $|y|=\epsilon_{i} M_{i}^{2}$, the only place to consider is $\partial^{\prime} \Sigma_{\lambda_{0}} \backslash B_{R_{1}}$, on which we have

$$
\begin{aligned}
& \partial_{3}\left(v_{i}(y)-\left(1-\epsilon_{0}\right)\left(1+c_{0}^{2}\right)^{-\frac{1}{2}}\left|y-e_{3}\right|^{-1}\right) \\
= & c_{i}\left(M_{i}^{-2} y+x_{i}^{\prime}\right) v_{i}^{3}(y)-\left(1-\epsilon_{0}\right)\left(1+c_{0}^{2}\right)^{-\frac{1}{2}}\left|y-e_{3}\right|^{-3} .
\end{aligned}
$$

Since $\lim _{i \rightarrow \infty} c_{i}\left(x_{i}^{\prime}\right) \leq 0$, (2.34) means that

$$
\partial_{3}\left(v_{i}(y)-\left(1-\epsilon_{0}\right)\left(1+c_{0}^{2}\right)^{-\frac{1}{2}}\left|y-e_{3}\right|^{-1}\right)<0
$$

at places where $v_{i}$ is close to $\left(1-\epsilon_{0}\right)\left(1+c_{0}^{2}\right)^{-\frac{1}{2}}\left|y-e_{3}\right|^{-1}$ on $\partial^{\prime} \Sigma_{\lambda_{0}} \backslash B_{R_{1}}$. This means that

$$
v_{i}>\left(1-\epsilon_{0}\right)\left(1+c_{0}^{2}\right)^{-\frac{1}{2}}\left|y-e_{3}\right|^{-1} \quad \text { on } \quad \partial^{\prime} \Sigma_{\lambda_{0}} \backslash B_{R_{1}} .
$$

Lemma 2.2 is established.

Once we have Lemma 2.2, the moving sphere process can start at $\lambda=\lambda_{0}$. Note that the equation for $w_{\lambda}+h_{\lambda}$ becomes

$$
\begin{cases}\left(\Delta+K_{i}\left(M_{i}^{-2} \cdot+x_{i}^{\prime}\right) \xi\right)\left(w_{\lambda}+h_{\lambda}\right) \leq 0 & \text { on } \Sigma_{\lambda}, \\ \left(\partial_{3}-c_{i}\left(M_{i}^{-2} \cdot+x_{i}^{\prime}\right) \xi\right)\left(w_{\lambda}+h_{\lambda}\right) \leq 0 & \text { on } \partial^{\prime} \Sigma_{\lambda} \cap \bar{O}_{\lambda} .\end{cases}
$$

This means that the maximum principle always holds for $w_{\lambda}+h_{\lambda}$ as long as it is positive on the boundary $\partial^{\prime \prime} \Omega_{i}$, which is certainly the case because $v_{i} \gg v_{i}^{\lambda}$ on $\partial^{\prime} \Omega_{i}$ and $h_{\lambda}$ is a perturbation. So the spheres can be moved to $\lambda_{1}=2\left(1+c_{0}^{2}\right)^{-\frac{1}{2}}$ to get a contradiction. 
2.1.3. Step three. In this subsection we deal with the case $\lim _{i \rightarrow \infty} c_{i}\left(x_{i}^{\prime}\right)=c_{0}>0$. $\bar{v}_{i}$ converges uniformly to $U$ in all finite subsets of $\left\{y \in \mathbb{R}^{3} ; y_{3} \geq-\lim _{i \rightarrow \infty} M_{i}^{2} x_{i 3}\right\}$. $U$ satisfies (still assuming $\lim _{i \rightarrow \infty} K_{i}\left(x_{i}\right)=3$ )

$$
\left\{\begin{array}{l}
\Delta U+3 U^{5}=0, \quad y_{3}>-\lim _{i \rightarrow \infty} M_{i}^{2} x_{i 3}, \\
\partial_{3} U=c_{0} U^{3}, \quad y_{3}=-\lim _{i \rightarrow \infty} M_{i}^{2} x_{i 3}, \\
U(0)=1 .
\end{array}\right.
$$

By Li and Zhu's classification theorem, $\lim _{i \rightarrow \infty} M_{i}^{2} x_{i 3}=c_{0}$ and

$$
U(y)=\left(1+|y|^{2}\right)^{-\frac{1}{2}} \text {. }
$$

Let

$$
v_{i}(y)=M_{i}^{-1} u_{i}\left(M_{i}^{-2} y+x_{i}^{\prime}\right)
$$

Then

$$
v_{i} \rightarrow U_{1}(y)=\left(1+\left|y-c_{0} e_{3}\right|^{2}\right)^{-\frac{1}{2}} .
$$

Let $v_{i}^{\lambda}$ be the Kelvin transformation of $v_{i}$. In this case we let

$$
\lambda_{0}=\frac{1}{2}\left(1+c_{0}^{2}\right)^{\frac{1}{2}}, \quad \lambda_{1}=2\left(1+c_{0}^{2}\right)^{\frac{1}{2}}
$$

because

$$
\begin{array}{ccc}
U_{1}>U_{1}^{\lambda} \quad \text { for } \quad \lambda \in\left(0,\left(1+c_{0}^{2}\right)^{\frac{1}{2}}\right), & |y|>\lambda, \\
\left.U_{1}<U_{1}^{\lambda} \text { for } \lambda>\left(1+c_{0}^{2}\right)^{\frac{1}{2}}\right), & |y|>\lambda .
\end{array}
$$

Let $w_{\lambda}=v_{i}-v_{i}^{\lambda}$. Then the equation for $w_{\lambda}$ is still described by (2.26) with $\xi_{1}$, $\xi_{2}, Q_{1}, Q_{2}$ defined as in step two and the estimates for $Q_{1}$ and $Q_{2}$ are still (2.27). Since $\lim _{i \rightarrow \infty} c_{i}\left(x_{i}^{\prime}\right)=c_{0}>0$, the construction of the test function $h_{\lambda}$ in this case is much more delicate.

The construction of $h_{\lambda}$ consists of two parts. First we shall construct $h_{1}$ to control the region close to $\partial B_{\lambda}$; then this function is connected smoothly to 0 . Clearly $h_{1}$ creates new difficulties at the regions where it is connected to 0 . Next we use $h_{2}$ to control the region far enough to $\partial B_{\lambda} . h_{2}$ is 0 in regions close to $\partial B_{\lambda}$ and becomes negative as $y$ is far away from $\partial B_{\lambda}$. A parameter of $h_{2}$ is chosen to be so large that $h_{2}$ not only controls the difficulties from $Q_{1}$ and $Q_{2}$, but also those from $h_{1}$. One delicate thing is that $h_{2}$ does not create new difficulties. By choosing all the parameters carefully we shall obtain the following properties for $h_{\lambda}$ :

$$
\begin{array}{ll}
\Delta h_{\lambda}+K_{i}\left(M_{i}^{-2} y+x_{i}^{\prime}\right) \xi_{1} h_{\lambda}+Q_{1} \leq 0 & \text { on } \Sigma_{\lambda}, \\
\partial_{3} h_{\lambda}-c_{i}\left(M_{i}^{-2} y+x_{i}^{\prime}\right) \xi_{2} h_{\lambda}+Q_{2} \leq 0 & \text { on } \partial \Sigma_{\lambda} \cap \partial \mathbb{R}_{+}^{3} \cap \overline{O_{\lambda}}, \\
h_{\lambda}=0 \quad \text { on } \partial B_{\lambda} \cap \mathbb{R}_{+}^{3}, & \\
h_{\lambda}=o(1)|y|^{-1} \quad \text { on } \Sigma_{\lambda} . &
\end{array}
$$

We first construct $h_{2}$. Let

$$
\phi(y)=1-A^{\frac{1}{4}}\left|y+r e_{3}\right|^{-\frac{1}{4}}, \quad r=|y|,
$$

where $A>5 \lambda_{1}$ is to be determined. Let $\Omega_{1} \subset \mathbb{R}_{+}^{3}$ be the region where $\phi$ is positive. Let $\Gamma_{1}$ be the 0 level surface of $\phi$. A direct computation shows that

$$
\mathbb{R}_{+}^{3} \backslash B(0,2 A) \subset \Omega_{1} \subset \mathbb{R}_{+}^{3} \backslash B(0, A / 3) .
$$

Let

$$
h_{2}(y)= \begin{cases}0 & \text { on } \mathbb{R}_{+}^{3} \backslash\left(\Omega_{1} \cup B_{\lambda}\right), \\ -C_{6} M_{i}^{-2} \phi^{2} & \text { on } \Sigma_{\lambda} \cap \Omega_{1},\end{cases}
$$


where $C_{6}$ is a large constant to be determined. From here we see that $h_{2}$ is $C^{1}$ across $\Gamma_{1}$. By a direct computation,

$$
\begin{aligned}
\Delta h_{2}= & -C_{6} M_{i}^{-2}\left(2|\nabla \phi|^{2}+2 \phi \Delta \phi\right) \\
= & -C_{6} M_{i}^{-2}\left(\frac{1}{8} A^{\frac{1}{2}}\left(2 r^{2}+2 r y_{3}\right)^{-\frac{9}{4}}\left(5 r^{2}+8 r y_{3}+3 y_{3}^{2}\right)\right. \\
& \left.+\frac{1}{2} \phi A^{\frac{1}{4}}\left(2 r^{2}+2 r y_{3}\right)^{-\frac{17}{8}}\left(\frac{3}{4} r^{2}+2 r y_{3}+\frac{5}{4} y_{3}^{2}\right)\right) \\
\leq & -\frac{C_{6}}{50} M_{i}^{-2} A^{\frac{1}{2}} r^{-\frac{5}{2}} \quad \text { on } \Omega_{1} \cap \Sigma_{\lambda} .
\end{aligned}
$$

From here we observe that $h_{2}$ is weakly superharmonic in $\Sigma_{\lambda}$ since $\nabla h_{2}=0$ on $\Gamma_{1}$. On $\partial \Omega_{1} \cap \partial \mathbb{R}_{+}^{3} \cap \overline{O_{\lambda}}$ we need to estimate

$$
\partial_{3} h_{2}-c_{i}\left(M_{i}^{-2} \cdot+x_{i}^{\prime}\right) \xi_{2} h_{2}+Q_{2}
$$

Since

$$
c_{i}\left(M_{i}^{-2} y+x_{i}^{\prime}\right) \xi_{2} \leq \frac{M}{1+r^{2}} \quad \text { on } \quad \overline{O_{\lambda}} \cap \partial \mathbb{R}_{+}^{3}
$$

for some $M>0$ depending on $c_{0}$, it is enough to show that

$$
\partial_{3} h_{2}-\frac{M}{1+r^{2}} h_{2}+Q_{2} \leq 0 \quad \text { on } \partial \Omega_{1} \cap \partial \mathbb{R}_{+}^{3} \cap \overline{O_{\lambda}} .
$$

By the definition of $h_{2}$, if $A\left(c_{0}\right)$ is large, we have

$$
\begin{aligned}
& \partial_{3} h_{2}-\frac{M}{1+r^{2}} h_{2}=-2 C_{6} M_{i}^{-2}\left(\partial_{3} \phi-\frac{M}{2\left(1+r^{2}\right)} \phi\right) \phi \\
\leq & -2 C_{6} M_{i}^{-2}\left(\partial_{3} \phi-\frac{M}{2\left(1+r^{2}\right)}\right) \phi \\
= & -2 C_{6} M_{i}^{-2}\left(\frac{1}{4} A^{\frac{1}{4}}\left(2 r^{2}\right)^{-\frac{9}{8}} r-\frac{M}{2\left(1+r^{2}\right)}\right) \phi \\
\leq & -\frac{C_{6}}{8} M_{i}^{-2} A^{\frac{1}{4}} r^{-\frac{5}{4}} \phi \quad \partial \Omega_{1} \cap \partial \mathbb{R}_{+}^{3} .
\end{aligned}
$$

Here $A$ is fixed. We observe that if $|y|>16 A, \phi(y)>\frac{1}{2}$. So by choosing $C_{6}$ large enough we have

$\left(\partial_{3}-c_{i}\left(M_{i}^{-2} \cdot+x_{i}^{\prime}\right) \xi_{2}\right) h_{2}+Q_{2} \leq-\frac{C_{6}}{16} M_{i}^{-2} A^{\frac{1}{4}} r^{-\frac{5}{4}} \quad$ on $\partial\left(\Sigma_{\lambda} \backslash B_{16 A}\right) \cap \partial \mathbb{R}_{+}^{3} \cap \overline{O_{\lambda}}$.

By (2.38) we also know

$$
\left(\partial_{3}-c_{i}\left(M_{i}^{-2} \cdot+x_{i}^{\prime}\right) \xi_{2}\right) h_{2} \leq 0 \quad \text { on } \partial\left(\Sigma_{\lambda} \cap B_{16 A}\right) \cap \partial \mathbb{R}_{+}^{3} \cap \overline{O_{\lambda}} .
$$

So the most important feature of $h_{2}$ is that it does not create new difficulties, even though it does not control all the error terms in the whole $\Sigma_{\lambda}$.

Now we define $h_{1}$ to be

$$
h_{1}(y)= \begin{cases}-C_{5} M_{i}^{-2}(r-\lambda) e^{N y_{3}} & \text { in } \Sigma_{\lambda} \cap B_{16 A}, \\ 0 & \text { in } \Sigma_{\lambda} \backslash B_{17 A}, \\ \text { smooth connection such that } h_{1} \leq 0 \text { in } & \text { in } \Sigma_{\lambda} \cap \overline{B_{17 A}} \backslash B_{16 A},\end{cases}
$$

where $C_{5}$ and $N$ are large positive constants to be determined. $A$ is the one in the definition of $h_{2}$, which has been determined. Also the "smooth connection" in the 
definition means that $\left|\nabla h_{1}(y)\right|,\left|\nabla^{2} h_{1}(y)\right| \leq C_{7}\left(C_{5}, A\right) M_{i}^{-2}$ for $16 A<|y|<17 A$, $y \in \Sigma_{\lambda}$.

By the definition of $h_{1}$,

$$
\begin{aligned}
\Delta h_{1}(y) & =-C_{5} M_{i}^{-2} e^{N y_{3}}\left(\frac{2}{r}+\frac{2 N}{r} y_{3}+N^{2}(r-\lambda)\right) \\
& \leq-2 C_{5} M_{i}^{-2} r^{-1}, \quad y \in B_{16 A} \cap \Sigma_{\lambda} .
\end{aligned}
$$

Here we require $C_{5}$ to satisfy

$$
\Delta h_{1}+Q_{1} \leq 0, \quad y \in B_{16 A} \cap \Sigma_{\lambda} .
$$

On the boundary we have

$$
\begin{aligned}
& \partial_{3} h_{1}-c_{i}\left(M_{i}^{-2} y^{\prime}+x_{i}^{\prime}\right) \xi_{2} h_{1} \\
& \quad=-C_{5} M_{i}^{-2}(r-\lambda)\left(N-c_{i}\left(M_{i}^{-2} y^{\prime}+x_{i}^{\prime}\right) \xi_{2}\right) \quad \text { on } \partial\left(\Sigma_{\lambda} \cap B_{16 A}\right) \cap \partial \mathbb{R}_{+}^{3} \cap \overline{O_{\lambda}} .
\end{aligned}
$$

So by choosing $N\left(c_{0}\right)$ large and $C_{5}$ larger if necessary we have

$$
\partial_{3} h_{1}-c_{i}\left(M_{i}^{-2} y^{\prime}+x_{i}^{\prime}\right) \xi_{2} h_{1}+Q_{2} \leq 0 \quad \text { on } \partial\left(\Sigma_{\lambda} \cap B_{16 A}\right) \cap \partial \mathbb{R}_{+}^{3} \cap \overline{O_{\lambda}} .
$$

So far $C_{5}, N$, and $A$ are all determined. We finally determine $C_{6}$. One last requirement for $C_{6}$ is to control the bad part of $h_{1}$ in $\left(B_{17 A} \backslash B_{16 A}\right) \cap \Sigma_{\lambda}$. By choosing $C_{6}$ larger if necessary we see from (2.38) and (2.39) that the errors caused by bending $h_{1}$ to 0 can be controlled by $h_{2}$. Therefore the first two equations of (2.35) are established. Here we also use the fact that $h_{\lambda} \leq 0$ in $\Sigma_{\lambda}$. By the definitions of $h_{1}$ and $h_{2}$, the other two equations of (2.35) are also satisfied. We are left with how to let the moving sphere process start. This part is similar to step two. We leave the details to the interested readers. Proposition 2.1 is established.

Proposition 2.1 is the major step in deriving the energy estimate in Corollary 2.1. Based on the previous works of Y. Y. Li 23, Han and $\mathrm{Li} 22$ and $\mathrm{Li}$ and Zhang [24, it is standard to derive Corollary 2.1. Therefore we only mention the major steps and the main idea in this argument. First one uses Schoen's selection process to find all large maximums of $u$ in, say, $B_{2 R}^{+}$. Around each of these local maximums there is a small neighborhood in which $u$ looks like a standard bubble which has most of its energy in it. The distance between these local maximums is the crucial information to find and this is the place where the Harnack inequality in Proposition 2.1 is used. The essential difference between this locally defined equation and those globally defined equations (such as the ones in [23]) is that one cannot find two bubbles closest to each other. For each bubble, there certainly exists a bubble closest to it, but one certainly cannot assume the first bubble is the closest one to the second bubble. This difficulty, which comes from the local nature of the equation, requires a different approach than those in 23. The way to overcome this difficulty is to rescale the equation so that after the scaling, the equation is centered at the first bubble and the distance between the first bubble and the second bubble is one. Then the Harnack inequality in Proposition 2.1 applied to the local region implies that the first bubble and the second bubble must have comparable magnitudes. Then it is possible to show that two bubbles cannot tend to the same blow-up point because otherwise a harmonic function with a positive second order term can be found. This second term will lead to a contradiction in the Pohozaev Identity. Note that the important second order term can only be proved to be positive if the adjacent bubbles have comparable magnitudes, which is the key information revealed by the Harnack inequality. Once we have known that 
all bubbles are far apart, it is possible to use standard elliptic estimates to show that the behavior of $u$ near each large local maximum is like a harmonic function with fast decay. So (2.5) as well as (2.6) can be obtained.

2.2. Harnack inequality in the interior of $M$. Now we consider $V \backslash N\left(\Gamma, \epsilon_{0}\right)$. Let $d$ be the distance between $V \backslash N\left(\Gamma, \epsilon_{0}\right)$ and $\partial M$. Over this interior region we don't need to assume $M$ to be locally conformally flat. We shall establish the following inequality:

Proposition 2.2. Let $p \in V \backslash N\left(\Gamma, \epsilon_{0}\right)$. There exist $\epsilon_{2}(M, g, \Lambda, d)>0$ and $C(M, g, \Lambda, d)>0$ such that

$$
\sup _{B\left(p, \epsilon_{2} / 3\right)} u \cdot \inf _{B\left(p, 2 \epsilon_{2} / 3\right)} u \leq C \epsilon_{2}^{-1} .
$$

The proof of Proposition 2.2 follows from the argument in [25]. Only a small modification is needed to adjust to the current situation. The outline is as follows. Suppose (2.45) does not hold. Then we can select $x_{i}$ as a sequence of local maximums of $u_{i}$. Then the equation can be written in conformal normal coordinates centered at $x_{i}$. After rescaling, $u_{i}$ becomes $v_{i}$, a sequence that converges in $C_{l o c}^{2}\left(\mathbb{R}^{3}\right)$ to a standard bubble whose maximum is 1 . Then consider the Kelvin transformation of $v_{i}(y): v_{i}^{\lambda}(y)$. By comparing the equation for $v_{i}$ and $v_{i}^{\lambda}$ we see that the only new term in this context is the term:

$$
\left(K_{i}\left(M_{i}^{-2} y^{\lambda}+x_{i}\right)-K_{i}\left(M_{i}^{-2} y+x_{i}\right)\right)\left(v_{i}^{\lambda}\right)^{5} \quad \text { in } \quad \Sigma_{\lambda}
$$

where $\Sigma_{\lambda}$ becomes a symmetric domain defined appropriately. The above term is of the harmless order $O\left(M_{i}^{-2}|y|^{-4}\right)$. By using the same test function we used in [25], a contradiction can be obtained correspondingly. We leave the details to the interested readers. Proposition 2.2 is proved.

Proposition 2.1 and Proposition 2.2 lead to Theorem 1.1 by the arguments in 24 and [26].

\section{Appendix}

In this section we provide some details of the Schoen selection process for the convenience of the readers. We only provide details for case one and case two. The details for case three are similar.

3.1. A calculus lemma. First we use a calculus lemma to simplify some computations. This calculus lemma was first used in [24].

Lemma 3.1. Let $u \in C^{0}\left(\overline{B_{1} \cap\{t>-T\}}\right), \quad T \geq 0$. Then for every $a>0$, there exists $x \in B_{1} \cap\{t \geq-T\}$ such that

$$
u(x) \geq \frac{1}{2^{a}} \max _{\bar{B}_{\sigma}^{T}(x)} u \quad \text { and } \quad \sigma^{a} u(x) \geq \frac{1}{2^{a}} u(0),
$$

where $B_{\sigma}^{T}(x)=B(x, \sigma) \cap\{t \geq-T\}, \sigma=(1-|x|) / 2$.

Proof of Lemma 3.1. First we remark that if $T \geq 1$, the selection is over the whole $B_{1}$. To prove the lemma, consider

$$
v(y)=(1-|y|)^{a} u(y)
$$

Let $x \in \bar{B}_{1}^{T}$ be a maximum point of $v$ and let $\sigma=(1-|x|) / 2$. By comparing $v(x)$ with $v(0)$ and $v(z)$ for all $z$ in $\bar{B}_{\sigma}^{T}(x)$, we see that $x$ and $\sigma$ have the desired properties. 
3.2. The selection process for case one in the proof of Proposition 2.1. In this subsection we explain why $x_{i}$ can be considered as a local maximum of $u_{i}$, assuming $u_{i}^{2}\left(x_{i}\right) x_{i 3} \rightarrow \infty$. We shall apply Lemma 3.1 for $T>1$; i.e., the selection is over the whole $B_{1}$. Let $r_{i}=\left|x_{i 3}\right| / 2$, so $u_{i}\left(x_{i}\right) r_{i}^{\frac{1}{2}} \rightarrow \infty$. Apply Lemma 3.1 to the function $u_{i}\left(x_{i}+r_{i} \cdot\right)$ over $B_{1}$. Then the conclusion can be translated as follows. There is $a_{i} \in B\left(x_{i}, r_{i}\right)$ such that

$$
u_{i}\left(a_{i}\right) \geq \max _{\bar{B}\left(a_{i}, \sigma_{i}\right)} 2^{-\frac{1}{2}} u_{i}(x),
$$

where $\sigma_{i}:=\frac{1}{2}\left(r_{i}-\left|a_{i}-x_{i}\right|\right)$ and

$$
\sigma_{i}^{\frac{1}{2}} u_{i}\left(a_{i}\right) \geq 2^{-\frac{1}{2}} u_{i}\left(x_{i}\right) r_{i}^{\frac{1}{2}} \rightarrow \infty .
$$

Let

$$
\tilde{v}_{i}(y)=u_{i}\left(a_{i}\right)^{-1} u_{i}\left(u_{i}\left(a_{i}\right)^{-2} y+a_{i}\right), \quad|y| \leq u_{i}\left(a_{i}\right)^{2} \sigma_{i}
$$

Then

$$
\tilde{v}_{i}(y) \leq 2^{\frac{1}{2}} \text { for }|y| \leq u_{i}\left(a_{i}\right)^{2} \sigma_{i} \rightarrow \infty
$$

Clearly $\tilde{v}_{i}$ satisfies

$$
\Delta \tilde{v}_{i}(y)+K_{i}\left(u_{i}\left(a_{i}\right)^{-2} y+a_{i}\right) \tilde{v}_{i}(y)^{5}=0, \quad|y| \leq u_{i}\left(a_{i}\right)^{2} \sigma_{i} .
$$

So by standard elliptic theory we know there is a subsequence of $\tilde{v}_{i}(y)$ (still denoted by $\left.\tilde{v}_{i}(y)\right)$ that converges uniformly to $\tilde{U}_{0}(y)$ on all compact subsets of $\mathbb{R}^{3}$. $\tilde{U}_{0}(y)$ satisfies

$$
\Delta \tilde{U}_{0}(y)+\tilde{K}_{0} \tilde{U}_{0}(y)^{5}=0, \quad y \in \mathbb{R}^{3},
$$

where $\tilde{K}_{0}=\lim _{i \rightarrow \infty} K_{i}\left(a_{i}\right)$. With no loss of generality we assume $\tilde{K}_{0}=3$. So by the well-known classification theorem of Caffarelli-Gidas-Spruck [8],

$$
\tilde{v}_{i}(y)=\left(\frac{\mu}{1+\mu^{2}|y-z|^{2}}\right)^{\frac{1}{2}}
$$

for some $z \in \mathbb{R}^{3}$ and $\mu \geq 1$. So $\tilde{U}_{0}(y)$ has an absolute maximum at $z$. Consequently $\tilde{v}_{i}(y)$ has a local maximum at $z_{i}$ close to $z$ when $i$ is large.

Let $\bar{x}_{i}=u_{i}\left(a_{i}\right)^{-2} z_{i}+a_{i}$. Then the $\left\{\bar{x}_{i}\right\}$ are local maximum points of $u_{i}$. Also it is easy to verify that

$$
u_{i}\left(\bar{x}_{i}\right) \geq \frac{\mu}{2} u_{i}\left(a_{i}\right) \geq \frac{\mu}{2} u\left(x_{i}\right)
$$

and $\bar{x}_{i} \in B\left(a_{i}, \sigma_{i}\right) \subset B\left(x_{i}, r_{i}\right)$, which means $\bar{x}_{i 3} \geq \frac{1}{2} x_{i 3}$. Consequently,

$$
u_{i}^{2}\left(\bar{x}_{i}\right) \bar{x}_{i 3} \rightarrow \infty \text {. }
$$

So we can consider $x_{i}$ as $\bar{x}_{i}$ at the beginning. The $\bar{v}_{i}(y)$ defined in (2.8) converge in $C_{l o c}^{2}\left(\mathbb{R}^{3}\right)$ to

$$
U(y)=\left(1+|y|^{2}\right)^{-\frac{1}{2}}
$$


3.3. Selection for case two in the proof of Proposition 2.1. In this subsection we show that $x_{i}$ can be considered as a local maximum of $u_{i}$ under the assumption $u_{i}^{2}\left(x_{i}\right) x_{i 3}=O(1)$.

By applying Lemma 3.1 to $u_{i}\left(x_{i}+\frac{1}{2} \cdot\right)$ with $a=1 / 2$ and $T=-x_{i 3}$ we can find $\bar{x}_{i} \in \overline{B\left(x_{i}, 1 / 2\right)} \cap \overline{\left\{x_{3}>-x_{i 3}\right\}}$ such that

$$
u_{i}\left(\bar{x}_{i}\right) \geq 2^{-\frac{1}{2}} \max u_{i} \quad \text { over } \overline{B\left(x_{i}, \sigma_{i}\right) \cap\left\{x_{3}>-x_{i 3}\right\}}
$$

and $\sigma_{i}^{\frac{1}{2}} u_{i}\left(\bar{x}_{i}\right) \geq 2^{-\frac{1}{2}} u_{i}\left(x_{i}\right)$, where $\sigma_{i}=\frac{1}{2}\left(\frac{1}{2}-\left|x_{i}-\bar{x}_{i}\right|\right)$. By these two inequalities we have

$$
u_{i}\left(\bar{x}_{i}\right) \geq u_{i}\left(x_{i}\right) \rightarrow \infty, \quad u_{i}\left(\bar{x}_{i}\right) \sigma_{i}^{\frac{1}{2}} \rightarrow \infty
$$

and $u_{i}\left(\bar{x}_{i}\right) \inf _{\partial B_{2}^{+}} u_{i} \geq i$. Now we can assume that $\bar{T}_{i}:=u_{i}^{2}\left(\bar{x}_{i}\right) \bar{x}_{i 3}$ is bounded because otherwise by replacing $x_{i}$ by $\bar{x}_{i}$ we go back to case one, which has been discussed. Let

$$
\hat{v}_{i}(y)=u_{i}\left(\bar{x}_{i}\right)^{-1} u_{i}\left(u_{i}\left(\bar{x}_{i}\right)^{-2} y+\bar{x}_{i}\right) .
$$

Then $\hat{v}_{i}$ satisfies

$$
\begin{cases}\Delta \hat{v}_{i}(y)+K_{i}\left(u_{i}\left(\bar{x}_{i}\right)^{-2} y+\bar{x}_{i}\right) \hat{v}_{i}(y)^{5}=0, & y \in \bar{\Omega}_{i}, \\ \partial_{3} \hat{v}_{i}(y)=c_{i}\left(u_{i}\left(\bar{x}_{i}\right)^{-2} y^{\prime}+\bar{x}_{i}^{\prime}\right) \hat{v}_{i}(y)^{3} & \text { on }\left\{y_{3}=-\bar{T}_{i}\right\} \cap \partial \bar{\Omega}_{i},\end{cases}
$$

where $\bar{\Omega}_{i}=\left\{y \mid u_{i}\left(\bar{x}_{i}\right)^{-2} y+\bar{x}_{i} \in B_{3}^{+}\right\}$. It is clear that the lower part of $\partial \bar{\Omega}_{i}$ is a subset of $\left\{y_{3}=-\bar{T}_{i}\right\}$. Moreover by the facts $u_{i}\left(\bar{x}_{i}\right) \bar{x}_{i 3}^{\frac{1}{2}} \leq C$ and $u_{i}\left(\bar{x}_{i}\right) \sigma_{i}^{\frac{1}{2}} \rightarrow \infty$ we know that $\hat{v}_{i}$ is uniformly bounded on all compact subsets of $\left\{y_{3} \geq-\lim _{i \rightarrow \infty} \bar{T}_{i}\right\}$. With no loss of generality we assume that $K_{i}\left(\bar{x}_{i}\right) \rightarrow 3$. Then by elliptic estimates, $\hat{v}_{i}\left(y-\bar{T}_{i} e_{3}\right)$ converges in the $C^{2}$ norm on all compact subsets of $\overline{\mathbb{R}}_{+}^{3}$ to $\bar{U}$, which solves

$$
\begin{cases}\Delta \bar{U}+3 \bar{U}^{5}=0 & \text { on } \mathbb{R}_{+}^{3}, \\ \partial_{3} \bar{U}=c \bar{U}^{3} & \text { for } y_{3}=0,\end{cases}
$$

where $c=\lim _{i \rightarrow \infty} c_{i}\left(\bar{x}_{i}^{\prime}\right)$. By Li and Zhu's classification result [27, we know that $\bar{U}$ has a unique maximum point $z_{0}$ in $\overline{\mathbb{R}_{+}^{3}}$. By the $C^{2}$ convergence of $\hat{v}_{i}\left(\cdot-\bar{T}_{i} e_{3}\right)$ to $\bar{U}$, we can find $\left\{y_{i}\right\}_{i=1,2, \ldots}$ as local maximum points of $\hat{v}_{i}\left(\cdot-\bar{T}_{i} e_{3}\right)$ that approach $z_{0}$ as $i \rightarrow \infty$. Then by the definition of $\hat{v}_{i}$, we know that $u_{i}\left(\bar{x}_{i}\right)^{-2}\left(y_{i}-\bar{T}_{i} e_{3}\right)+\bar{x}_{i}$ are local maximum points of $u_{i}$. So we redefine $x_{i}$ as $u_{i}\left(\bar{x}_{i}\right)^{-2}\left(y_{i}-\bar{T}_{i} e_{3}\right)+\bar{x}_{i}$. So the $\bar{v}_{i}$ defined in (2.8) converge to the function $U_{1}$ in (2.10).

\section{REFERENCES}

[1] A. Ambrosetti, Y. Y. Li, A. Malchiodi, On the Yamabe problem and the scalar curvature problems under boundary conditions. Math. Ann. 322 (2002), no. 4, 667-699. MR1905107 (2003e:53038)

[2] H. Araújo, Existence and compactness of minimizers of the Yamabe problem on manifolds with boundary. Comm. Anal. Geom. 12 (2004), no. 3, 487-510. MR2128601(2005m:53052)

[3] T. Aubin, Équations différentielles non linéaires et problème de Yamabe concernant la courbure scalaire, J. Math. Pures Appl. 55 (1976), 269-296. MR0431287 (55:4288)

[4] M. Berti, A. Malchiodi, Non-compactness and multiplicity results for the Yamabe problem on $S^{n}$. J. Funct. Anal. 180 (2001), no. 1, 210-241. MR1814428(2002b:53049)

[5] S. Brendle, A generalization of the Yamabe flow for manifolds with boundary. Asian J. Math. 6 (2002), no. 4, 625-644. MR1958085 (2003m:53052)

[6] S. Brendle, Blow-up phenomena for the Yamabe equation, J. Amer. Math. Soc. 24 (2008), no 4, 951-979.

[7] S. Brendle, F. C. Marques, Blow-up phenomena for the Yamabe PDE II, preprint 2007. 
[8] L. Caffarelli, B. Gidas, J. Spruck, Asymptotic symmetry and local behavior of semilinear equations with critical Sobolev growth, Comm. Pure Appl. Math. 42 (1989), 271-297. MR982351 (90c:35075)

[9] L. Caffarelli, A Harnack inequality approach to the regularity of free boundaries. I. Lipschitz free boundaries are $C^{1, \alpha}$. Rev. Mat. Iberoamericana 3 (1987), no. 2, 139-162. MR990856 (90d:35306)

[10] L. Caffarelli, A Harnack inequality approach to the regularity of free boundaries. II. Flat free boundaries are Lipschitz. Comm. Pure Appl. Math. 42 (1989), no. 1, 55-78. MR973745 (90b:35246)

[11] C. C. Chen, C. S. Lin, Estimates of the conformal scalar curvature equation via the method of moving planes, Comm. Pure Appl. Math. 50 (1997), 971-1017. MR1466584 (98k:35051)

[12] C. C. Chen, C. S. Lin, Estimate of the conformal scalar curvature equation via the method of moving planes. II. J. Differential Geom. 49 (1998), no. 1, 115-178. MR1642113(2000h:35045)

[13] C. C. Chen, C. S. Lin, Prescribing scalar curvature on $S^{N}$. I. A priori estimates. J. Differential Geom. 57 (2001), no. 1, 67-171. MR.1871492(2002j:53038)

[14] Z. Djadli, A. Malchiodi, M. O. Ahmedou, Prescribing scalar and boundary mean curvature on the three dimensional sphere, J. Geom. Anal. 13 (2003), no. 2, 255-289. MR1967027 (2004d:53032)

[15] O. Druet, Compactness for Yamabe metrics in low dimensions. Int. Math. Res. Not. 2004, no. 23, 1143-1191. MR2041549 (2005b:53056)

[16] O. Druet, Compactness for Yamabe metrics in low dimensions. Int. Math. Res. Not. 2004, no. 23, 1143-1191. MR2041549 (2005b:53056)

[17] J. Escobar, Conformal deformation of a Riemannian metric to a constant scalar curvature metric with constant mean curvature on the boundary. Indiana Univ. Math. J. 45 (1996), no. 4, 917-943. MR 1444473 (98d:53051)

[18] J. Escobar, Conformal deformation of a Riemannian metric to a scalar flat metric with constant mean curvature on the boundary. Ann. of Math. (2) 136 (1992), no. 1, 1-50. MR1173925 (93e:53046)

[19] J. Escobar, The Yamabe problem on manifolds with boundary. J. Differential Geom. 35 (1992), no. 1, 21-84. MR1152225 (93b:53030)

[20] V. Felli, M. Ould Ahmedou, Compactness results in conformal deformations of Riemannian metrics on manifolds with boundaries. Math. Z. 244 (2003), no. 1, 175-210. MR1981882 (2004d:53034)

[21] M. Khuri, F.C. Marques, R. Schoen, A compactness theorem for the Yamabe problem, prepint, 2007.

[22] Z. Han, Y. Y. Li, The Yamabe problem on manifolds with boundary: Existence and compactness results. Duke Math. J. 99 (1999), no. 3, 489-542. MR.1712631 (2000j:53045)

[23] Y. Y. Li, Prescribing scalar curvature on $S^{n}$ and related problems, Part I, J. Diff. Equations 120 (1995), 319-410. MR.1347349 (98b:53031)

[24] Y. Y. Li, L. Zhang, Liouville type theorems and Harnack type inequalities for semilinear elliptic equations, J. Anal. Math, 90(2003), 27-87. MR.2001065 (2004i:35118)

[25] Y.Y. Li, L. Zhang, A Harnack type inequality for the Yamabe equation in low dimensions, Calc. Var. Partial Differential Equations 20 (2004), 133-151. MR2057491 (2005f:53053)

[26] Y.Y. Li, L. Zhang, Compactness of solutions to the Yamabe problem II, Calc. Var. Partial Differential Equations, 24 (2005), no. 2, 185-237. MR2164927(2006f:53049)

[27] Y. Y. Li, M. Zhu, Uniqueness theorems through the method of moving spheres, Duke Math. J. 80, No. 2 (1995), 383-417. MR1369398 (96k:35061)

[28] F. C. Marques, Existence results for the Yamabe problem on manifolds with boundary. Indiana Univ. Math. J. 54 (2005), no. 6, 1599-1620. MR2189679 (2006j:53047)

[29] F. C. Marques, A priori estimates for the Yamabe problem in the non-locally conformally flat case. J. Differential Geom. 71 (2005), no. 2, 315-346. MR2197144 (2006i:53046)

[30] M. Ould Ahmedou, On the prescribed scalar and zero mean curvature on 3-dimensional manifolds with umbilic boundary. Adv. Nonlinear Stud. 6 (2006), no. 1, 13-46. MR2196889 (2007b:53074)

[31] R. Schoen, Courses at Stanford University, 1988, and New York University, 1989.

[32] R. Schoen, Variational theory for the total scalar curvature functional for Riemannian metrics and related topics, in "Topics in Calculus of Variations", Lecture Notes in Mathematics, Vol. 1365 (M. Giaquinta, Ed.), Springer-Verlag, Berlin/New York, 1989. MR994021 (90g:58023) 
[33] K. Taira, The Yamabe problem and nonlinear boundary value problems. J. Differential Equations 122 (1995), no. 2, 316-372. MR.1355895 (96g:53050)

[34] S. Taliaferro, L. Zhang, Asymptotic symmetry for conformal scalar curvature equation with singularities, Calc. Var. Partial Differential Equations 26 (2006), no. 4, 401-428. MR 2235880 (2007d:35105)

[35] N. Trudinger, Remarks concerning the conformal deformation of Riemannian structures on compact manifolds, Ann. Scuola Norm. Sup. Cl. Sci. (3) 22 (1968), 265-274. MR0240748 (39:2093)

[36] H. Yamabe, On a deformation of Riemannian structures on compact manifolds, Osaka Math. J. 12 (1960), 21-37. MR0125546 (23:A2847)

[37] L. Zhang, Refined asymptotic estimates for conformal scalar curvature equations via moving sphere method, J. Funct. Anal, 192 (2002), 491-516. MR.1923411 (2003j:35113)

Department of Mathematics, University of Alabama at Birmingham, 1300 University Boulevard, 452 Campbell Hall, Birmingham, Alabama 35294-1170

E-mail address: leizhang@math.uab.edu 